TRANSACTIONS OF THE

AMERICAN MATHEMATICAL SOCIETY

Volume 353, Number 12, Pages 5003-5027

S 0002-9947(01)02661-7

Article electronically published on April 11, 2001

\title{
REPLACING MODEL CATEGORIES WITH SIMPLICIAL ONES
}

\author{
DANIEL DUGGER
}

\begin{abstract}
In this paper we show that model categories of a very broad class can be replaced up to Quillen equivalence by simplicial model categories.
\end{abstract}

\section{INTRODUCTION}

1.1. Summary. Model categories were introduced by Quillen $Q$ as a very general setting in which one can 'do homotopy theory'. Among them is a distinguished class called the simplicial model categories, which are essentially characterized by the following property: certain formulae which in an arbitrary model category hold only up to homotopy, in simplicial model categories they actually hold on the nose. Actually, such formulae can be made to hold on the nose in an arbitrary model category, but only at the expense of carefully making choices; what's useful about simplicial model categories is that they come with a canonical set of choices already made. This turns out to be quite a convenience, and simplicial model categories are much easier to work with than arbitrary ones.

It is certainly not true that all model categories of interest are simplicial, but many of them happen to be. It is natural to wonder if there is a significant difference between simplicial and non-simplicial model categories. The obvious question is, what do we mean by 'significant'?

Now Quillen also introduced a notion of equivalence between model categories, which involves having a pair of adjoint functors satisfying certain conditions. Two model categories which are 'Quillen equivalent' represent the same underlying homotopy theory. The purpose of this note is to show that a very large class of model categories are Quillen equivalent to simplicial ones. In other words, for this class of model categories there is no significant difference between simplicial and non-simplicial, at least as far as the underlying homotopy theory is concerned.

More explicitly, if $M$ is a model category, then one can look at the category of simplicial objects $s M$. This is also a model category in a standard way -it has a so-called Reedy model structure, which is usually not simplicial. There is also an obvious pair of adjoint functors

$$
c_{*}: M \rightleftarrows s M: e v_{0} .
$$

Here $c_{*}$ (the left-adjoint) associates to $X \in M$ the discrete simplicial object consisting of $X$ in every dimension, and $e v_{0}$ sends $Z_{*} \in s M$ to its zeroth object $Z_{0}$. Under broad conditions on the model category $M$, we describe a way of making

Received by the editors December 8, 1998 and, in revised form, December 1, 1999.

2000 Mathematics Subject Classification. Primary 55U35.

(C)2001 American Mathematical Society 
$s M$ into a simplicial model category with the property that the above functors are a Quillen equivalence.

To describe the conditions which we assume on $M$, it is necessary to recall the technique of localization. This is the process by which one adds weak equivalences to a model category while keeping the cofibrations the same; in recent years it has become recognized as a fundamental tool in homotopy theory. While it is not true that all model categories can be localized, at the moment there are two known large classes which can be: these are the left proper, cellular model categories of Hirschhorn [ $\mathrm{H}$ ] and the left proper, combinatorial model categories of Smith [S]. Cellular model categories include topological spaces, diagrams of topological spaces, simplicial sets, the various model structures on bisimplicial sets, and many more. Combinatorial model categories include practically any model category of 'algebraic' origin, and in particular anything built up in some way from diagrams of simplicial sets. (There is a large overlap between the two classes.)

We'll use the following acronyms for the two types of model categories which will appear in the paper:

(LpCe): Left proper, cellular;

(LpCo): Left proper, combinatorial.

A precise definition of these terms is available in section 2.

At this point we have introduced enough terminology to state our main result:

Theorem 1.2. Let $M$ be a model category of type ( $\mathrm{LpCe}$ ) or ( $\mathrm{LpCo}$ ). Then there exists a simplicial model category structure on $s M$ for which the adjoint functors $c_{*}: M \rightleftarrows s M: e v_{0}$ are a Quillen equivalence.

The model structure is defined by saying that a map is a

- weak equivalence if it is a hocolim-equivalence (defined below);

- cofibration if it is a Reedy cofibration;

- fibration if it has the right-lifting-property with respect to the trivial cofibrations.

We remark that a very similar result has been obtained independently by Rezk, Schwede, and Shipley [RSS. Their result applies in particular to model categories which are cofibrantly-generated and stable (meaning essentially that the suspension functor is an equivalence on the homotopy category), although they also realized that their arguments would probably apply to the cellular case. The motivation behind the stability assumption will be explained in the last section of this paper.

We close the introduction by saying a little about the proof of Theorem 1.2 The key point is that the Reedy model structure on $s M$ satisfies all the axioms of a simplicial model category except one. This last axiom is satisfied if one looks only at maps between simplicial resolutions - these are objects of $s M$ which are Reedy fibrant and have the property that every face and degneracy map is a weak equivalence. Our approach is to produce a new model structure on $s M$ in which the simplicial resolutions become the fibrant objects, which allows us to replace any object by a simplicial resolution up to weak equivalence. Since the simplicial structure is well-behaved on simplicial resolutions, this essentially implies that it is well-behaved everywhere (and left properness is a key step in concluding this).

The model structure which does all this is produced by localization, and is an instance of a more general 'hocolim model structure' for diagram categories. Let $M$ be a model category and $\mathcal{C}$ be a small indexing category. Given a diagram 
$X: \mathcal{C} \rightarrow M$ we may form its homotopy colimit by choosing a framing and applying the usual Bousfield-Kan formula (see $[\overline{\mathrm{DHK}}]$ or $[\underline{\mathrm{H}}]$ ), but this only has homotopy meaning when $X$ is a diagram of cofibrant objects. For the purposes of this paper, we define the corrected homotopy colimit to be the object obtained by first replacing each object of $X$ with a (functorial) cofibrant-replacement, and then taking the usual homotopy colimit of the resulting diagram. A map $X \rightarrow Y$ between diagrams is a hocolim-equivalence if it induces a weak equivalence between the corrected homotopy colimits.

When $M$ is cofibrantly-generated-which holds in particular if $M$ is of type (LpCe) or (LpCo) - then the category of diagrams $M^{\mathcal{C}}$ has a Bousfield-Kan model structure: the weak equivalences (resp. fibrations) are maps of diagrams which are weak equivalences (resp. fibrations) at each spot. Our second main result is the following:

Theorem 1.3. Let $M$ be a model category of type (LpCe) or (LpCo), and let $\mathcal{C}$ be a small category whose nerve is contractible. Then there is a model structure on $M^{\mathcal{e}}$ in which

(i) the weak equivalences are the hocolim-equivalences;

(ii) the cofibrations are the Bousfield-Kan cofibrations;

(iii) the fibrations are the maps with the appropriate right-lifting-property.

Moreover, the fibrant objects of $M^{\mathrm{C}}$ are the objectwise fibrant diagrams which are 'homotopically-constant'-i.e., in which every map of the diagram is a weak equivalence.

It might be hoped that this model category will be useful in answering problems about homotopy colimits other than just the ones encountered in this paper.

1.4. Organization of the paper. Section 2 is a review of localization theory for model categories. The section also gives brief definitions of the terms 'cellular' and 'combinatorial'.

Section 3 recalls Quillen's notion of a simplicial model category. We prove some basic simplifications of the axioms, whose upshot is that in left proper model categories the hard parts of Quillen's axioms can be checked just on the fibrant objects.

Section 4 starts by recalling that $s M$ has a Reedy model category structure, as well as a 'categorical' simplicial action. The purpose of the section is to investigate how the two relate to each other. We prove that almost all of Quillen's axioms for a simplicial model category are satisfied, but not all of them. We also show that if one looks only at maps between simplicial resolutions, then all the axioms are satisfied. Most of this material has strong precedents in Hirschhorn $[\mathrm{H}$, although the results we need are not quite proven there.

In section 5 we produce our 'hocolim model structure' on diagram categories, and in particular prove Theorem 1.3 above. There are versions of this result for both the Bousfield-Kan and Reedy model category structures on diagrams. Although the Reedy version is the one which is ultimately shown to be simplicial, we need to use the Bousfield-Kan version to prove the basic properties of the Reedy one.

Section 6 proves the main theorem, that the Reedy version of the hocolimstructure is simplicial, and section 7 lists some questions which may be of further interest. 
Finally, an appendix is enclosed in which we prove some basic facts about model categories needed elsewhere in the paper. The appendix should be regarded as a supplement to the books $[\mathrm{H}]$ and $[\mathrm{Ho}]$.

1.5. Notation and terminology. We assume the reader is familiar with the language and basic results of model categories - the most complete reference for this is Hirschhorn $[\mathrm{H}]$, and we try to follow his conventions closely. Hovey's book [Ho] also contains a wealth of material, and is very accessible.

Since the paper by nature is concerned with model categories which may not be simplicial, it is necessary for us to work with the machinery of framings and function complexes. Recall that in a model category one has the notion of a cylinder object for $X$, which is an object which 'looks like' $X \times \Delta^{1}$. A cosimplicial framing is a massive generalization of this: it is a way of assigning to each object $X$ a cosimplicial object which 'looks like' $[n] \mapsto X \times \Delta^{n}$. In the same vein, a simplicial framing assigns to each object $Z$ a simplicial object which 'looks like' $[n] \mapsto Z^{\Delta^{n}}$. These can be used to define the homotopy function complex from $X$ to $Z$, which is a simplicial set that acts as the replacement of the mapping space $[n] \mapsto H o m\left(X \times \Delta^{n}, Z\right)$. The reader will find a detailed treatment of all this in $[\mathrm{H}]$ and [Ho, Chapter 5], and we will assume a familiarity with this machinery.

If $\mathcal{C}$ is a category and $X, Y$ are objects in $\mathcal{C}, \mathcal{C}(X, Y)$ denotes the set of maps from $X$ to $Y$ in $\mathcal{C}$. If $M$ is a model category and $X$ and $Y$ are objects, $\underline{\mathcal{N}}(X, Y)$ denotes a homotopy function complex from $X$ to $Y$.

Various tensor products and exponentials are used throughout the paper, and there is some potential for confusion here. If $X: \mathcal{C} \rightarrow \mathcal{D}$ is a $\mathcal{C}$-diagram in $\mathcal{D}$ and $K: \mathrm{C}^{o p} \rightarrow$ Set is a $\mathrm{C}^{o p}$-diagram of sets, we use $X \otimes \mathrm{e} K$ to denote the resulting coend (cf. $\mathrm{McL})$. Likewise, if $L: \mathcal{C} \rightarrow$ Set, then $\operatorname{hom}^{\mathrm{C}}(L, X)$ denotes the resulting end.

On the other hand, if $T$ is a category with a simplicial action (see section 2 for a definition) and $X \in T, K \in$ SSet, then $X \otimes K$ and $X^{K}$ denote the tensor and hom functors associated to the action. It should always be clear from context whether we are talking about coends or simplicial actions.

1.6. Acknowledgements. It was Stefan Schwede who had the idea that something like all this might work, and I would like to thank him for sharing his ideas. Jeff Smith generously explained his work on combinatorial model categories to me, and I also had helpful conversations with Phil Hirschhorn and Dan Kan.

\section{Localization of Model Categories}

This section contains background material on localization, together with brief definitions of cellular and combinatorial model categories. Many readers will want to skip ahead, and only refer back to this section as needed.

Let $M$ be a model category and let $S$ be a set of maps in $M$. The process of localizing $M$ at $S$ involves producing a new model category structure on $M$ by adding the elements of $S$ to the class of weak equivalences. Because of the model category axioms, this may force one into regarding other maps as weak equivalences as well (for instance, retracts of elements of $S$ ). Bousfield was the first to develop a systematic approach to this problem, which we recall next. 


\section{Definition 2.1.}

(a) An $S$-local object of $M$ is a fibrant object $X$ such that for every map $A \rightarrow B$ in $S$, the induced map of homotopy function complexes $\underline{\mathcal{M}}(B, X) \rightarrow \underline{\mathcal{M}}(A, X)$ is a weak equivalence of simplicial sets.

(b) An $S$-local equivalence is a map $A \rightarrow B$ with the property that $\underline{\mathcal{M}}(B, X) \rightarrow$ $\underline{\mathcal{M}}(A, X)$ is a weak equivalence for every $S$-local object $X$.

In other words, the $S$-local objects are the ones which see every map in $S$ as if it were a weak equivalence. The $S$-local equivalences are those maps which are seen as weak equivalences by every $S$-local object. The idea is that the $S$-local equivalences are the maps which are forced into being weak equivalences as soon as we expand our notion of weak equivalence to include the maps in $S$.

Definition 2.2. An $S$-localization of $M$ is a model category $L_{S} M$ with the properties that

(a) the underlying category of $L_{S} M$ is $M$;

(b) the cofibrations in $L_{S} M$ are the same as those in $M$;

(c) the weak equivalences in $L_{S} M$ are the $S$-local equivalences.

Hirschhorn $[\underline{\mathrm{H}}]$ refers to these as 'left Bousfield localizations' of $M$, but we will just call them localizations (or $S$-localizations). An $S$-localization need not always exist. If it does exist, it is clearly unique. The fibrant objects in $L_{S} M$ will be precisely the $S$-local objects, but the fibrations may be somewhat mysterious.

There are two main classes of model categories where $S$-localizations are always known to exist (for any set of maps $S$ ). These are the left proper, cellular model categories of Hirschhorn [H], and the left proper, combinatorial model categories of Smith [S]. The next item on the agenda is to recall the definitions of these classes.

Definition 2.3. A model category $M$ is left proper if the pushout of a weak equivalence along a cofibration is again a weak equivalence. That is, if $A \rightarrow B$ is a weak equivalence and $A \rightarrow C$ is a cofibration, then in the pushout square

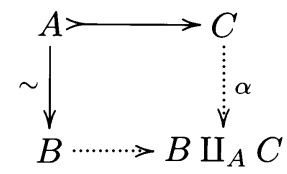

one has that the map $\alpha$ is also a weak equivalence.

Definition 2.4. A model category is called combinatorial if it is cofibrantlygenerated and if the underlying category is locally presentable.

The defintion of locally presentable will not be recalled here. It is a technical, category-theoretic condition which ensures that every object is small (with respect to some cardinal), and so the small object argument can be made for any set of maps. The reader may consult [B] for a precise definition.

Definition 2.5. A model category $M$ is called cellular if it is cofibrantly-generated and has a set $I$ of generating cofibrations and a set $J$ of generating trivial cofibrations such that

(a) the domains and codomains of the maps in $I$ are compact relative to $I$;

(b) the domains of the elements of $J$ are small relative to $I$;

(c) every cofibration in $M$ is an effective monomorphism. 
This definition is quite technical, and we will not make matters worse by recalling the precise meanings of 'compact' and 'effective monomorphism'. The reader is free to look these up in $[\mathrm{H}]$. Again, the point of the definition is to guarantee that the small object argument can be applied to any collection of cofibrations.

We'll use the following acronyms for the two types of model categories considered in this paper:

(LpCe): Left proper, cellular;

(LpCo): Left proper, combinatorial.

The only things the reader will need to know about these classes are that

1. a model category in the class may be localized at any set of maps, and the resulting model category is again in the class;

2. if $M$ is in the class and $\mathcal{C}$ is an indexing category, then the Bousfield-Kan model structure on the diagram category $M^{\mathcal{C}}$ also belongs to the class;

3 . if $M$ is in the class and $\mathcal{C}$ is a Reedy category, then the Reedy model structure on $M^{\mathcal{C}}$ is also in the class.

(The Bousfield-Kan and Reedy model structures on diagram categories are recalled in section 5 , and are treated in detail both in $[\mathrm{H}]$ and $[\mathrm{Ho}$.) These basic properties are consequences of the work of Hirschhorn $[\mathrm{H}]$ and Smith [S].

\section{Simplicial Model Categories}

This section consists mainly of preliminaries. We recall Quillen's notion of a simplicial model category, and prove some basic results which simplify what is needed to verify the axioms. The most important point is that for left proper model categories, Quillen's axiom SM7 can be verified essentially by showing that the simplicial structure behaves well on fibrant objects.

Recall that a simplicial action on a category $\mathcal{C}$ consists of three functors

$$
\begin{aligned}
\operatorname{Map}(X, Y) \in \text { sSet } & \text { for } X, Y \in \mathcal{C}, \\
X \otimes K \in \mathcal{C} & \text { for } X \in \mathcal{C} \text { and } K \in \text { sSet }, \\
X^{K} \in \mathcal{C} & \text { for } X \in \mathcal{C} \text { and } K \in \text { sSet }
\end{aligned}
$$

such that certain familiar axioms hold. (This is also called a Cartesian closed simplicial action in some circles.)

A model category $M$ with a simplicial action is called a simplicial model category if the axiom below is satisfied:

(SM7) If $f: A \longmapsto B$ is a cofibration in $M$ and $j: K \longmapsto L$ is a cofibration in $s$ Set, then the natural map

$$
f \otimes j:(A \otimes L) \coprod_{A \otimes K}(B \otimes K) \longrightarrow B \otimes L
$$

has the following properties:

(i) It is a cofibration;

(ii) If $f: A \longmapsto B$ is a trivial cofibration, so is $f \otimes j$;

(iii) If $j: K \longmapsto L$ is a trivial cofibration, so is $f \otimes j$.

These statements are equivalent to the following dual versions. It will be necessary for us to go back and forth between these equivalent formulations, and we'll be very cavalier about doing this. 
(SM7, Dual Version) If $p: X \longmapsto Y$ is a fibration in $M$ and $j: K \longmapsto L$ is a cofibration in $s$ Set, then the natural map

$$
\operatorname{hom}(j, p): X^{L} \rightarrow X^{K} \times_{Y^{K}} Y^{L}
$$

has the following properties:

(i) If $p$ is trivial fibration, then so is $\operatorname{hom}(j, p)$;

(ii) $\operatorname{hom}(j, p)$ is a fibration;

(iii) If $j: K \longmapsto L$ is a trivial cofibration, then $\operatorname{hom}(j, p)$ is a trivial fibration.

Remark 3.1. It is useful to know that once SM7(i) has been checked, (ii) reduces to verifying that if $f: A \stackrel{\sim}{\longmapsto} B$ is a trivial cofibration and $J \in s$ Set, then $A \otimes J \rightarrow B \otimes J$ is a weak equivalence. This follows from looking at the diagram

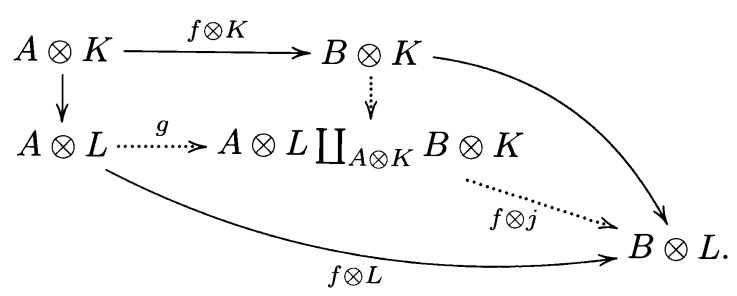

If $f \otimes K$ is a trivial cofibration, its pushout $g$ is a trivial cofibration. But then if $f \otimes L$ is also known to be a trivial cofibration, $f \otimes j$ must be a weak equivalence by the two-out-of-three property.

When SM7 is difficult to verify, it is because the objects $A \otimes K$ and $X^{K}$ may not behave well unless $A$ is cofibrant and $X$ is fibrant. SM7 only talks about maps behaving well under these operations, and this can be troublesome to check when one has no control over the domains and targets. The following proposition says that in certain circumstances (notably left properness) one may verify parts of SM7 by only checking how the simplicial action behaves on fibrant objects.

Proposition 3.2. Let $M$ be a model category with a simplicial action.

(a) If SMY(i) holds, then SM7(ii) is equivalent to requiring that for every simplicial set $K$ the functors $(-)^{K}$ preserve fibrations between fibrant objects;

(b) If $S M r(i)$ and (ii) both hold, and if in addition $M$ is left proper, then (iii) may be reduced to the condition that if $Z \in M$ is fibrant and $K \longmapsto L$ a trivial cofibration in sSet, then $Z^{L} \rightarrow Z^{K}$ is a weak equivalence.

Proof. The simplicial action gives adjoint functors

$$
(-) \otimes K: M \rightleftarrows M:(-)^{K} \text {. }
$$

If SM7(i) holds, then the right adjoint preserves trivial fibrations. On the other hand, Remark 3.1 says that verifying SM7(ii) reduces to showing that the right adjoint preserves fibrations. This in turn is equivalent to the condition that $(-)^{K}$ preserves fibrations between fibrant objects by Corollary A.2 from the appendix. This proves (a).

For (b), let $p: X \rightarrow Y$ be a fibration in $M$ and $j: K \rightarrow L$ a trivial cofibration in $s$ Set. We need to check that $X^{L} \rightarrow X^{K} \times_{Y^{K}} Y^{L}$ is a trivial fibration, and by a theorem of Kan about left proper model categories, it suffices to check the rightlifting-property with respect to cofibrations $f: A \rightarrow B$ between cofibrant objects. 
By using adjointness, this is equivalent to checking that the maps $f \otimes j$ are all trivial cofibrations.

To check that $f \otimes j$ is a weak equivalence, we will look at its image under the functor $M\left(-, Z_{*}\right)$ where $Z_{*}$ is Reedy fibrant. The induced map is

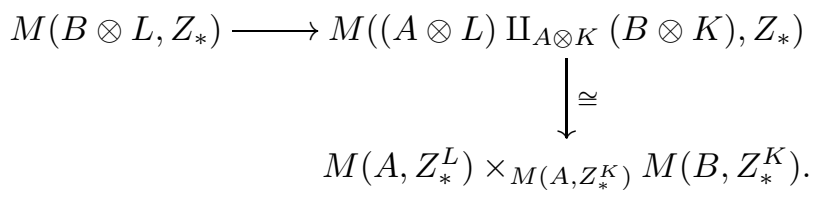

(Here $Z_{*}^{L}$ denotes the simplicial object $i \mapsto\left(Z_{i}\right)^{L}$.) Now let $\alpha$ denote $Z_{*}^{L} \rightarrow Z_{*}^{K}$ and consider the diagram

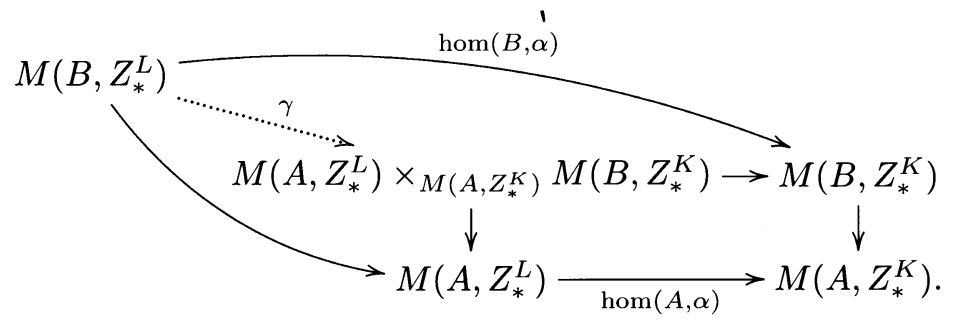

Our hypotheses guarantee that $\alpha$ is a Reedy trivial fibration in $s M$. Since $A$ and $B$ are cofibrant, the maps $\operatorname{hom}(A, \alpha)$ and $\operatorname{hom}(B, \alpha)$ are therefore trivial fibrations. The map $\beta$ is a pullback of $\operatorname{hom}(A, \alpha)$, so it too is a trivial fibration. Then the two-out-of-three property gives that the map labelled $\gamma$ is a weak equivalence.

So we have shown that every Reedy fibrant $Z_{*} \in s M$ gives a weak equivalence $M\left(B \otimes L, Z_{*}\right) \rightarrow M\left((A \otimes L) \amalg_{A \otimes K}(B \otimes K), Z_{*}\right)$. By Proposition A.1 it follows that $(A \otimes L) \amalg_{A \otimes K}(B \otimes K) \rightarrow B \otimes L$ is a weak equivalence. This is what was wanted.

\section{Simplicial Structures and Reedy Model Categories}

Here we recall that if $M$ is a category with limits and colimits, then $s M$ has a 'categorical' simplicial action which is very much like the natural simplicial action on $s$ Set. When $M$ is a model category, $s M$ also has a 'Reedy model category structure' - but this turns out not to be compatible with the simplicial action. In other words, $s M$ does not become a simplicial model category in this way. This failure, however, is not as bad as it could be; we verify that two-thirds of SM7 are actually satisfied. In the later sections of this paper, the game will be to modify the Reedy model structure so that it actually becomes simplicial. This is possible because of a surprising property of the Reedy model category: the final third of SM7 is satisfied if one only looks at maps between simplicial resolutions.

4.1. Categorical simplicial structures. If $T$ is a category which is both complete and co-complete, then the category $s T$ admits a certain 'categorical' simplicial action. This was studied by Quillen in [Q, Chapter 2], however he only wrote down a simple formula for the tensor product. It is crucial for our purposes to have a manageable formula for the exponential as well; luckily, this is not hard.

First some general notation: 
(i) For a set $S$ and an object $X \in T$, let $X \cdot S$ denote $\coprod_{S} X$. That is, $X \cdot S$ is a coproduct of copies of $X$, one for each element of $S$.

(ii) Likewise, $X^{\cdot S}$ denotes $\prod_{S} X$ (note the dot in the superscript).

(iii) Given a small category $\mathcal{C}$ and functors $X: \mathcal{C} \rightarrow T, K: \mathcal{C}^{o p} \rightarrow$ Set, let $X \otimes_{\mathfrak{e}} K$ denote the resulting coend:

$$
X \otimes_{\mathfrak{e}} K:=\operatorname{coeq}\left(\coprod_{c \rightarrow d \in \mathcal{e}} X c \cdot K d \longrightarrow \coprod_{c \in C} X c \cdot K c\right) .
$$

(iv) Given $X: \mathcal{C} \rightarrow T$ and $K: \mathcal{C} \rightarrow \operatorname{Set}$, let $\operatorname{hom}^{\mathrm{e}}(K, X)$ denote the resulting end:

$$
\operatorname{hom}^{\mathrm{e}}(K, X):=\mathrm{eq}\left(\prod_{c \in C} X c^{\cdot K c} \longrightarrow \prod_{c \rightarrow d \in \mathcal{C}} X d^{\cdot K c}\right) \text {. }
$$

These constructions allow us to describe the simplicial action on $s T$ : for $X \in s T$ and $K \in$ sSet,

(i) $X \otimes K$ is the simplicial object with $X_{n} \cdot K_{n}$ in degree $n$, and the obvious face and degeneracy maps;

(ii) $X^{K}$ is the simplicial object which in degree $n$ is $\operatorname{hom}^{\Delta^{o p}}\left(K \times \Delta^{n}, X\right)$, with the face and degeneracy maps induced by the cofaces and codegeneracies of $\Delta^{*}$;

(iii) $\operatorname{Map}(X, Y)$ is the simplicial set whose $n$-simplices are $s T\left(X \otimes \Delta^{n}, Y\right)$.

The verification that these definitions do indeed equip $s T$ with a simplicial action will be left to the reader. We do, however, wish to isolate the following simple lemma which will be needed later:

\section{Lemma 4.2.}

(a) For $X \in s T, \operatorname{hom}^{\Delta^{o p}}\left(\Delta^{n}, X\right) \cong X_{n}$.

(b) For $X \in s T$ and $K, L \in s \mathcal{S} e t$, $\operatorname{hom}^{\Delta^{o p}}\left(L, X^{K}\right) \cong \operatorname{hom}^{\Delta^{o p}}(K \times L, X)$.

Proof. The proof of (a) is a direct computation. To prove (b), first note that the functors $X^{(-)}$and hom $^{\Delta^{o p}}(-, X)$ both transform colimits to limits - this follows from adjointness considerations. Since any simplicial set is a colimit of $\Delta^{n}$ 's, we reduce at once to the case $K=\Delta^{n}$ and $L=\Delta^{m}$. But this case follows directly from the definitions, together with part (a).

4.3. The Reedy model structure on $s M$. Let $M$ be a model category. We briefly recall the Reedy model structure on the category of simplicial objects $s M$. This is described in more detail in $[\mathrm{Ho}$ Theorem 5.2.5] and in $[\mathrm{H}]$.

If $X \in s M$, the nth matching object of $X$ is the object $M_{n} X$ defined by

$$
M_{n} X:=\operatorname{hom}^{\Delta^{o p}}\left(\partial \Delta^{n}, X\right) .
$$

(In what follows, we will tend to drop the $\Delta^{o p}$ from the superscript.) Note that the inclusion $\partial \Delta^{n} \rightarrow \Delta^{n}$ gives a canonical map $X_{n}=\operatorname{hom}\left(\Delta^{n}, X\right) \rightarrow \operatorname{hom}\left(\partial \Delta^{n}, X\right)=$ $M_{n} X$. If $f: X \rightarrow Y$ is a map between simplicial objects, one defines the $n t h$ matching map of $f$ to be the map

$$
X_{n} \longrightarrow M_{n} X \times_{M_{n} Y} Y_{n}
$$

We recall that $s M$ has a Reedy model category structure characterized by the following properties: 
(i) A map $X \rightarrow Y$ is a weak equivalence if each $X_{n} \rightarrow Y_{n}$ is a weak equivalence in $M$.

(ii) A map $X \rightarrow Y$ is a fibration (resp. trivial fibration) if each matching map $X_{n} \rightarrow M_{n} X \times_{M_{n} Y} Y_{n}$ is a fibration (resp. trivial fibration) in $M$.

With only a little more effort, it is possible to also give a concise description of the Reedy cofibrations - we will not need this, however.

The following proposition and remark explain how close the Reedy model structure on $s M$ comes to being simplicial.

Proposition 4.4. Let $M$ be a model category. The Reedy model structure on sM with the categorical simplicial action satisfies parts (i) and (ii) of SM7.

Proof. In this instance it is easier to prove SM7 in its dual version.

To check that $\alpha$ is a fibration or trivial fibration, we must check the same for the various matching maps of $\alpha$. Given a map of simplicial objects $Z \rightarrow W$, the matching map in dimension $n$ is

$$
\operatorname{hom}\left(\Delta^{n}, Z\right) \longrightarrow \operatorname{hom}\left(\partial \Delta^{n}, Z\right) \times_{\operatorname{hom}\left(\partial \Delta^{n}, Y\right)} \operatorname{hom}\left(\Delta^{n}, Y\right) .
$$

By using Lemma 4.2 and some mucking around, the matching map of $\alpha$ may be identified with

$$
\operatorname{hom}\left(L \otimes \Delta^{n}, X\right) \longrightarrow \operatorname{hom}\left(H_{n}, X\right) \times_{\operatorname{hom}\left(H_{n}, Y\right)} \operatorname{hom}\left(L \otimes \Delta^{n}, Y\right)
$$

where $H_{n}=\left(L \otimes \partial \Delta^{n}\right) \coprod_{K \otimes \partial \Delta^{n}}\left(K \otimes \Delta^{n}\right)$. Since $H_{n} \rightarrow L \otimes \Delta^{n}$ is a cofibration of simplicial sets (because $s \delta e t$ is a simplicial model category), we are reduced to proving the lemma below.

Lemma 4.5. If $X \rightarrow Y$ is a Reedy fibration (resp. trivial fibration) in $s M$ and $K \longmapsto L$ is a cofibration in sSet, then

$$
\operatorname{hom}(L, X) \longrightarrow \operatorname{hom}(K, X) \times \operatorname{hom}(K, Y) \operatorname{hom}(L, Y)
$$

is a fibration (resp. trivial fibration) in $M$.

Proof. By standard techniques, we can immediately reduce to the case where $K \longmapsto$ $L$ is the map $\partial \Delta^{n} \rightarrow \Delta^{n}$. But then this is just the characterization of Reedy fibrations and trivial fibrations given above.

Remark 4.6. It is certainly not true that the Reedy structure satisfies SM7(iii). An easy counterexample is obtained by taking any Reedy cofibrant $A \in s M$ and looking at one of the maps $A \otimes \Delta^{0} \rightarrow A \otimes \Delta^{1}$. In simplicial degree 0 this looks like $A_{0} \rightarrow A_{0} \amalg A_{0}$, and so in particular the map is not an objectwise weak equivalence.

Stefan Schwede has had the idea to localize at the set of maps

$$
(A \otimes L) \coprod_{A \otimes K}(B \otimes K) \longrightarrow B \otimes L
$$

so as to force SM7(iii) to hold. The first difficulty is that these maps obviously form a proper class, and the various localization machines in place require one to be localizing a set of maps. This is fixed without much trouble: if $M$ is cofibrantlygenerated, then $s M$ is as well (see $[\mathbf{H}]$ ), and it turns out to be enough to localize at the above maps when $K \rightarrow L$ is $\partial \Delta^{n} \rightarrow \Delta^{n}$ and $A \longmapsto B$ is a generating cofibration.

There is a second difficulty which is more subtle. By localizing a model category one introduces additional trivial cofibrations, so we must go back and re-prove SM7(ii) for the localized category. In many cases this would be inaccessible, because 
one usually doesn't have much hold on what the new weak equivalences are. An important fact about our approach is that we can completely identify these.

Although SM7(iii) does not hold in the Reedy model category, a limited version does hold - the statement is true as long as all the domains and targets of our maps are simplicial resolutions. We won't need the full power of this result, but only the limited version stated in the proposition below. In some sense this is the key fact which makes the whole paper work; in the next section we will produce a model structure for which the simplicial resolutions are precisely the fibrant objects, and SM7 will reduce to checking that the simplicial structure behaves well on these.

Definition 4.7. A simplicial resolution is a Reedy fibrant object $X \in s M$ with the property that every face and degeneracy map is a weak equivalence.

Proposition 4.8. Let $P \in s M$ be a simplicial resolution, and let $K \stackrel{\sim}{\rightarrow} L$ be a trivial cofibration of simplicial sets. Then $P^{L} \rightarrow P^{K}$ is a Reedy trivial fibration between simplicial resolutions.

Proof. We already know the map in question is a fibration, by SM7(ii) for the Reedy model category $s M$ (Proposition 4.4). We show it is an objectwise weak equivalence. In dimension $m$ the map is

$$
\operatorname{hom}^{\Delta^{o p}}\left(L \times \Delta^{m}, P\right) \rightarrow \operatorname{hom}^{\Delta^{o p}}\left(K \times \Delta^{m}, P\right) .
$$

Since $K \times \Delta^{m} \rightarrow L \times \Delta^{m}$ is a trivial cofibration of simplicial sets, we reduce to proving the following:

$(*)$ If $I \rightarrow J$ is a trivial cofibration in $s$ Set, then $\operatorname{hom}^{\Delta^{o p}}(J, P) \rightarrow \operatorname{hom}^{\Delta^{o p}}(I, P)$ is a weak equivalence in $M$.

By standard techniques, this reduces to the case where $I \rightarrow J$ has the form $\Delta^{0} \rightarrow \Delta^{n}$. But hom $\left(\Delta^{0}, P\right)$ and $\operatorname{hom}\left(\Delta^{n}, P\right)$ may be canonically identified with $P_{0}$ and $P_{n}$, and the map $P_{n} \rightarrow P_{0}$ is a weak equivalence precisely because $P$ is a simplicial resolution. This shows that $P^{L} \rightarrow P^{K}$ is a Reedy trivial fibration.

The final step is to prove that $P^{K}$ and $P^{L}$ are simplicial resolutions. The same proof of course works for both.

Note first that $P^{K}$ must be Reedy fibrant, by SM7(ii) for the Reedy model category. We must show that every face and degeneracy is a weak equivalence. To do this, recall that $P^{K}$ is the simplicial object $[n] \mapsto \operatorname{hom}^{\Delta^{o p}}\left(K \times \Delta^{n}, P\right)$. The result labelled $(*)$ above, together with what we already know, says that the functor $\operatorname{hom}^{\Delta^{o p}}(-, P):$ s Set $\rightarrow M$ takes cofibrations to fibrations and trivial cofibrations to trivial fibrations. By a standard fact about model categories, it follows that weak equivalences between cofibrant objects must map to weak equivalences. In particular, the maps

$$
\operatorname{hom}^{\Delta^{o p}}\left(K \times \Delta^{n}, P\right) \rightarrow \operatorname{hom}^{\Delta^{o p}}\left(K \times \Delta^{m}, P\right)
$$

induced by some $\Delta^{n} \rightarrow \Delta^{m}$ are all weak equivalences. This is what we wanted.

\section{A Hocolim Model Structure on Diagrams}

In this section we produce certain model structures on diagram categories $M^{\mathfrak{C}}$, where a map is a weak equivalence provided it induces a weak equivalence between the corrected homotopy colimits. These model category structures are obtained 
as localizations, so our results are limited to the cases when $M$ is of type (LpCe) or $(\mathrm{LpCo})$. There are versions of the model structure corresponding both to the usual Bousfield-Kan (= objectwise fibration) and to the Reedy model categories of diagrams. In the case where $\mathrm{C}=\Delta^{o p}$, the Reedy version of this hocolim-structure will be shown in the next section to be simplicial and Quillen equivalent to $M$. The Bousfield-Kan version will not be used elsewhere in the paper, but we need it here as a stepping-stone in establishing the basic properties of the Reedy version.

If $M$ is a model category and $X: \mathcal{C} \rightarrow M$ is a diagram ( $\mathcal{C}$ a small indexing category), one can define the homotopy colimit of $X$ by-for instance - choosing a cosimplicial framing on $M$ and writing down an explicit formula (see $[\mathrm{H}]$ ). This definition has the slight drawback that it gives the 'correct' homotopy type only when the diagram $X$ consists of cofibrant objects. An objectwise weak equivalence $X \rightarrow Y$ induces a weak equivalence hocolim $X \stackrel{\sim}{\longrightarrow}$ hocolim $Y$ only when the entries of $X$ and $Y$ are all cofibrant.

For the purposes of this paper, we remedy the situation by defining the corrected homotopy colimit "corhocolim $X$ " to be the result of first replacing every object of $X$ by a (functorial) cofibrant replacement, and then taking the usual homotopy colimit of the resulting diagram. With this definition it is now true that any objectwise weak equivalence $X \rightarrow Y$ induces a weak equivalence corhocolim $X \rightarrow$ corhocolim $Y$. (The length of the name 'corhocolim' is only justified by the fact that we won't actually have to use it very often.)

Definition 5.1. A map of diagrams $X \rightarrow Y$ is said to be a hocolim-equivalence if the induced map corhocolim $X \rightarrow \operatorname{corhocolim} Y$ is a weak equivalence in $M$.

Note that every objectwise weak equivalence is a hocolim-equivalence.

Recall that when $M$ is a cofibrantly-generated model category, the category of diagrams $M^{\mathcal{C}}$ may be given a 'Bousfield-Kan' model structure: a map of diagrams $X \rightarrow Y$ is a weak equivalence (resp. fibration) if the maps $X_{c} \rightarrow Y_{c}$ are weak equivalences (resp. fibrations) for every $c \in \mathcal{C}$. (As always, the reader is referred to [H] for a detailed treatment.) Our goal will be to localize the Bousfield-Kan structure by inverting the hocolim-equivalences. Unfortunately, these form a proper class of maps, so our first task is to identify a set of maps such that inverting them is enough.

Assume now that $M$ is left proper and cofibrantly-generated. Proposition A.5 from the appendix says that there is a set $W$ of cofibrant objects with the property that a map $X \rightarrow Y$ is a weak equivalence if and only if it induces weak equivalences on function complexes $\underline{\mathcal{M}}(A, X) \rightarrow \underline{\mathcal{M}}(A, Y)$ for every $A \in W$.

Let $\mathcal{C}$ be a small category. For $i \in \mathcal{C}$ and $X \in M$, let $F_{i}^{X}$ denote the free diagram generated by $X$ at spot $i$. This is the diagram defined by

$$
F_{i}^{X}(j)=\coprod_{\mathfrak{e}(i, j)} X
$$

In other words, the object at spot $j$ in $F_{i}^{X}$ is a coproduct of $X$ 's, one for each map $i \rightarrow j$. Note that the functor $X \mapsto F_{i}^{X}$ is the left adjoint to $D \mapsto D_{i}$ : if $D$ is any diagram, then $M^{\mathcal{C}}\left(F_{i}^{X}, D\right)=M\left(X, D_{i}\right)$.

For every map $i \rightarrow j$ in $\mathcal{C}$ and $A \in W$, there are maps of diagrams

$$
F_{j}^{A} \rightarrow F_{i}^{A} .
$$

Let $S$ denote the set of all such maps. 
If $M$ is of type (LpCe) or (LpCo), then so is the diagram category $\left(M^{\mathfrak{C}}\right)_{B K}$. In particular, we may consider its localization at the set $S$; denote this localized model category by $S^{-1}\left(M^{\mathcal{E}}\right)_{B K}$. The goal of this section is the following:

Theorem 5.2. Assume that the nerve of $\mathcal{C}$ is contractible. Then:

(a) The weak equivalences in the localized model category $S^{-1}\left(M^{\mathfrak{E}}\right)_{B K}$ are precisely the hocolim-equivalences.

(b) The adjoint functors colim : $S^{-1}\left(M^{\mathcal{C}}\right)_{B K} \rightleftarrows M: c_{*}$ are a Quillen equivalence.

(c) The fibrant objects in $S^{-1}\left(M^{\mathcal{C}}\right)_{B K}$ are the objectwise fibrant diagrams with the property that every map in the diagram is a weak equivalence.

Remark 5.3. One might interpret this result in the following way. A diagram in $M^{\mathfrak{C}}$ morally has two kinds of homotopy invariants: it has homotopy invariants coming from the model category $M$, and homotopy invariants coming from the structure of the indexing category $\mathcal{C}$. The theorem says that if $\mathcal{C}$ is contractible and one localizes away all the homotopy invariants coming from $\mathcal{C}$, then all the remaining invariants factor through the homotopy colimit functor.

We need the following key result due to Chachólski and Scherer [CS] (also proven by Kan $[\overline{\mathrm{DHK}}]$ ):

Proposition 5.4. Let $M$ be a model category and let $\mathcal{C}$ be a small category whose nerve is contractible. If $X: \mathcal{C} \rightarrow M$ is an objectwise cofibrant diagram in which every map is a weak equivalence, then the natural maps $X_{\alpha} \rightarrow$ hocolim $X$ are also weak equivalences.

Corollary 5.5. Let $M$ and $\mathcal{C}$ be as above, but assume $M$ is cofibrantly-generated. Let $X \in M^{\mathcal{C}}$ be a cofibrant diagram in which every map is a weak equivalence. Then for any fibrant replacement $Z$ for corhocolim $X$, there exists a map of diagrams $X \rightarrow c_{*} Z$ which is an objectwise weak equivalence.

Proof. The adjunction colim: $M^{\mathfrak{C}} \rightleftarrows M: c_{*}$ is a Quillen pair when $M^{\mathfrak{C}}$ is given the Bousfield-Kan model structure. Then $\operatorname{colim} X$ is cofibrant and $\operatorname{colim} X \simeq$ corhocolim $X \simeq Z$, so there is a weak equivalence $\operatorname{colim} X \stackrel{\sim}{\longrightarrow} Z$ in $M$. Let $X \rightarrow c_{*} Z$ be the adjoint of this map. Since this map is a hocolim-equivalence by construction, the Chachólski-Scherer result shows that it must actually be an objectwise weak equivalence.

Proof of Theorem 5.2, We first prove (c). General localization machinery tells us that a diagram $X$ is fibrant in the localized model category iff $X$ is fibrant in $\left(M^{\mathfrak{C}}\right)_{B K}$ and the induced maps of homotopy function complexes

$$
\underline{M}^{\mathcal{C}}\left(F_{i}^{A}, X\right) \rightarrow \underline{M}^{\mathcal{C}}\left(F_{j}^{A}, X\right)
$$

are weak equivalences, for every $i \rightarrow j$ in $\mathcal{C}$ and every $A \in W$. We claim that in general the function complex $\underline{M}^{\mathcal{C}}\left(F_{i}^{A}, X\right)$ may be identified up to weak equivalence with the function complex $\mathcal{\mathcal { A }}\left(A, X_{i}\right)$; this follows by first using a simplicial resolution of $X$, and then using adjointness. Granting this, the above maps are weak equivalences precisely when the maps

$$
\underline{\mathcal{N}}\left(A, X_{i}\right) \rightarrow \underline{\mathcal{M}}\left(A, X_{j}\right)
$$

are also. But we choose $W$ so that this happens precisely when $X_{i} \rightarrow X_{j}$ is a weak equivalence. This proves (c). 
To prove (a), it will be enough to show that a map $A \rightarrow B$ between cofibrant objects is a hocolim-equivalence precisely when it is an $S$-local equivalence. This is because for $A$ and $B$ arbitrary, we can pick a cofibrant replacement in $\left(M^{\mathfrak{C}}\right)_{B K}$

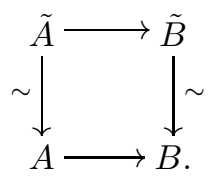

The vertical maps are objectwise weak equivalences, and therefore $\tilde{A} \rightarrow \tilde{B}$ is an $S$-local equivalence (resp. hocolim-equivalence) if and only if $A \rightarrow B$ is one. So if it is known that the two types of weak equivalence agree for maps between cofibrants, they must agree in general.

So now assume that $A$ and $B$ are cofibrant. Let $X$ be an $S$-local object, and let $Z$ be a fibrant replacement for corhocolim $X$. By the above corollary, there is a map of diagrams $X \rightarrow c_{*} Z$ which is an objectwise weak equivalence between fibrant objects. Consider the diagram

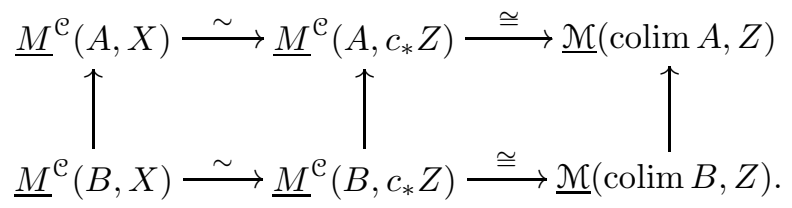

Here the horizontal arrows on the right are obtained by computing the function complexes using simplicial resolutions of the second variable, which allows us to use the adjunction between colim and $c_{*}$. The important point is that the maps are weak equivalences, not that they are isomorphisms.

Now the map $A \rightarrow B$ is an $S$-local equivalence provided that for every $S$-local object $X$, the map $\underline{M}^{\mathcal{C}}(B, X) \rightarrow \underline{M}^{\mathcal{C}}(A, X)$ is a weak equivalence. The above diagram essentially shows that this is the same as requiring that $\underline{\mathcal{N}}(\operatorname{colim} B, Z) \rightarrow$ $\underline{\mathcal{N}}(\operatorname{colim} A, Z)$ be a weak equivalence for every fibrant $Z \in M$. This in turn is equivalent to colim $A \rightarrow \operatorname{colim} B$ being a weak equivalence in $M$. Since the colimit functor is the 'correct' homotopy colimit for cofibrant diagrams, the last condition is precisely that $A \rightarrow B$ be a hocolim-equivalence.

For (b), first note that the adjoint functors do indeed form a Quillen pair; for instance, $c_{*}$ clearly preserves fibrations and trivial fibrations. Let $A$ be a cofibrant diagram and $Z \in M$ be a fibrant object. We must verify that a map colim $A \rightarrow Z$ is a weak equivalence iff the adjoint $A \rightarrow c_{*} Z$ is a hocolim-equivalence. Consider the commutative square

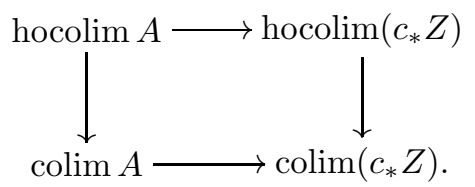

Note that $\operatorname{colim}\left(c_{*} Z\right) \cong Z$, since the indexing category is contractible (so in particular, connected). The result will follow once we show that the vertical maps are weak equivalences, because then the top map will be a weak equivalence precisely when the bottom map is - this is what was wanted. 
The left vertical map is a weak equivalence because $A$ is cofibrant. For the right map, pick some $i \in \mathcal{C}$ and consider the composable arrows

$$
Z=\left(c_{*} Z\right)_{i} \rightarrow \operatorname{hocolim} c_{*} Z \rightarrow \operatorname{colim} c_{*} Z \cong Z .
$$

Proposition 5.4 says that the first map is an equivalence. Since the composite is an actual isomorphism, the second map is a weak equivalence as well.

5.6. A hocolim structure for Reedy model categories. If $\mathcal{C}$ is a Reedy category, then there is another model structure on $M^{\mathcal{C}}$ called the Reedy model category structure. (In the cases where $\mathcal{C}$ is either $\Delta^{o p}$ or $\Delta$, these are the model structures on $s M$ and $c M$ discussed in section 2.) Reedy model structures have the advantage that they exist quite generally, not just under the assumption that $M$ is cofibrantly-generated. When one does have cofibrant-generation, the identity maps give a Quillen equivalence

$$
L:\left(M^{\mathcal{C}}\right)_{B K} \rightleftarrows\left(M^{\mathcal{C}}\right)_{\text {Reedy }}: R .
$$

The goal in this section is to localize the Reedy model structure at the hocolimequivalences. In the case where $\mathcal{C}=\Delta^{o p}$, this model category will be the one used to prove our main result, Theorem 1.2

The result we're after is the following:

Theorem 5.7. If the nerve of the Reedy category $\mathcal{C}$ is contractible, then

(a) The weak equivalences in $S^{-1}\left(M^{\mathcal{C}}\right)_{\text {Reedy }}$ are the hocolim-equivalences;

(b) The identity maps give a Quillen equivalence

$$
S^{-1}\left(M^{\mathcal{C}}\right)_{B K} \rightleftarrows S^{-1}\left(M^{\mathcal{C}}\right)_{\text {Reedy }}
$$

(c) The fibrant objects of $S^{-1}\left(M^{\mathcal{C}}\right)_{\text {Reedy }}$ are the the Reedy fibrant diagrams having the property that every map is a weak equivalence.

Proof. The proof of (c) is exactly the same as for the Bousfield-Kan structure. We next prove (a).

A general result of Hirschhorn $[\mathrm{H}]$ says that if $L: M \rightleftarrows N: R$ is a Quillen pair between localizable model categories, and $S$ is a set of maps in $M$, then the adjunction extends to a Quillen pair $S^{-1} M \rightleftarrows(L S)^{-1} N$. So in particular, in our case the identity maps give a Quillen pair

$$
S^{-1}\left(M^{\mathcal{C}}\right)_{B K} \rightleftarrows S^{-1}\left(M^{\mathcal{C}}\right)_{\text {Reedy }}
$$

Let $A \rightarrow B$ be a map of diagrams, and let $\bar{A} \rightarrow \bar{B}$ be a fibrant replacement in $S^{-1}\left(M^{\mathcal{C}}\right)_{B K}$. In other words, we have a square

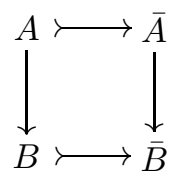

where the horizontal maps are Bousfield-Kan cofibrations and hocolim-equivalences. Because of the Quillen pair above, we know that these maps are also trivial cofibrations in $S^{-1}\left(M^{\mathcal{C}}\right)_{\text {Reedy }}$; in particular, they are Reedy $S$-local equivalences. 
Now let $\hat{A} \rightarrow \hat{B}$ be a fibrant replacement for $\bar{A} \rightarrow \bar{B}$ in the (unlocalized) Reedy model structure. This gives us a diagram

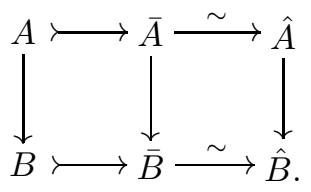

The right horizontal maps are objectwise weak equivalences, and the left horizontal maps are both hocolim-equivalences and Reedy $S$-local equivalences. Moreover, $\hat{A}$ and $\hat{B}$ are fibrant in both $S^{-1}\left(M^{\mathcal{C}}\right)_{B K}$ and $S^{-1}\left(M^{\mathcal{E}}\right)_{\text {Reedy }}$ : they are Reedy fibrant (hence objectwise fibrant) and have the property that every map in the diagram is a weak equivalence, because $\bar{A}$ and $\bar{B}$ have this property by construction.

The above diagram gives us everything we need. If $A \rightarrow B$ is a hocolimequivalence, then going around the diagram shows that $\hat{A} \rightarrow \hat{B}$ must be one as well. But these are fibrant objects in $S^{-1}\left(M^{\mathfrak{E}}\right)_{B K}$, and an $S$-local equivalence between fibrant objects is necessarily an objectwise weak equivalence. Then going back around the diagram, we find that $A \rightarrow B$ is also a Reedy $S$-local equivalence.

A similar case shows that if $A \rightarrow B$ is a Reedy $S$-local equivalence, it must be a hocolim-equivalence. In other words, the notions of Reedy $S$-local equivalence and hocolim-equivalence are identical.

Finally, the Quillen equivalence in part (b) follows from an immediate verification, using (a).

Remark 5.8. Hirschhorn has also shown that if $L: M \rightleftarrows N: R$ is a Quillen equivalence between localizable model categories, and $S$ is a set of maps in $M$, then the Quillen pair $L: S^{-1} M \rightleftarrows(L S)^{-1} N: R$ is also a Quillen equivalence. This gives another proof of part (b) of the corollary, but unfortunately it doesn't seem to give (a) as an immediate consequence. One appears to still need a proof such as the one we gave above.

\section{A simplicial model Structure on $s M$}

The goal of this section is to prove Theorem 1.2. Most of the work has been done already, and the proof is just a matter of assembling the details.

Let $M$ be a model category of type (LpCe) or ( $\mathrm{LpCo})$. We showed in the last section that there is a model structure on $s M$ in which a map $X \rightarrow Y$ is a

(i) weak equivalence if it is a hocolim-equivalence;

(ii) cofibration if it is a Reedy cofibration;

(iii) fibration if it has the right-lifting-property with respect to all trivial cofibrations.

Denote this model category by $s M_{h c}$.

Theorem 6.1. The categorical simplicial structure makes $s M_{h c}$ into a simplicial model category, for which the usual adjoint pair $c_{*}: M \rightleftarrows s M_{h c}: e v_{0}$ is a Quillen equivalence.

Remark 6.2. The above model structure was called the 'Reedy hocolim-model structure' in the last section, to constrast it with the analagous Bousfield-Kan hocolimstructure. The latter model category is definitely not simplicial, for the following reason: 
Assume for the moment that it is. Then given a trivial fibration $X \rightarrow Y$ in $S^{-1}(s M)_{B K}$ (which are exactly the trivial fibrations in $s M_{B K}$ ) it will also be the case that

$$
X^{\Delta^{n}} \rightarrow X^{\partial \Delta^{n}} \times_{Y^{\partial \Delta^{n}}} Y^{\Delta^{n}}
$$

is a trivial fibration. In particular, the map of zeroth-objects will be a trivial fibration in $M$. But a little thought shows that this map of zeroth spaces is exactly the $n$th matching map of $X \rightarrow Y$, and so we are claiming that $X \rightarrow Y$ is a Reedy trivial fibration. In other words, every trivial fibration in $s M_{B K}$ is a Reedy trivial fibration. It is easy to check that this is false.

Proof of Theorem 6.1. It is well known (and easy to check) that the functor $c_{*}$ maps cofibrations to Reedy cofibrations, and trivial cofibrations to Reedy trivial cofibrations. This is the statement that

$$
c_{*}: M \rightleftarrows s M_{\text {Reedy }}: e v_{0}
$$

is a Quillen pair. It follows at once that the same functors give a Quillen pair with $s M_{\text {Reedy }}$ replaced by $s M_{h c}$ (essentially because the identity maps give Quillen functors $\left.L: s M_{\text {Reedy }} \rightleftarrows s M_{h c}: R\right)$.

To show this is a Quillen equivalence, let $A \in M$ be cofibrant and $X \in s M_{h c}$ be fibrant (so that $X$ is a simplicial resolution). Let $\tilde{A}$ and $\tilde{X}$ denote the result of applying a functorial cofibrant replacement functor (in $M$ ) to $A$, and levelwise to $X$. Consider the diagram

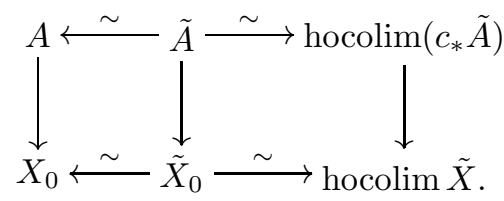

The right horizontal arrows are weak equivalences by Proposition 5.4 , since all the face and degeneracy maps of both $c_{*} \tilde{A}$ and $\tilde{X}$ are weak equivalences. So it follows that the right-most vertical map is a weak equivalence precisely when the left-most map is; i.e., $c_{*} A \rightarrow X$ is a hocolim-equivalence iff $A \rightarrow X_{0}$ is a weak equivalence in $M$. This establishes the Quillen equivalence.

Now we turn to the simplicial structure. Part (i) of SM7 follows from the corresponding result about $s M_{\text {Reedy }}$ (Proposition 4.4), because the cofibrations in the hocolim- and Reedy model categories are the same.

To prove (ii), it suffices to show that the functors $(-)^{K}$ preserve fibrations between fibrant objects (Proposition 3.2). Recall that in a localized model category, a map between fibrant (i.e. local) objects is a fibration if and only if it was a fibration in the un-localized model structure (see $[\underline{\mathrm{H}}]$ for a proof). So a fibration between fibrant objects in $s M_{h c}$ is precisely a Reedy fibration between simplicial resolutions. We know that $(-)^{K}$ preserves Reedy fibrations by SM7(ii) for the Reedy model category (4.4), and $(-)^{K}$ takes simplicial resolutions to simplicial resolutions by Proposition 4.8 .

Finally, we must prove SM7(iii). Since $M$ is left proper, the same is true for $s M_{\text {Reedy }}$ and therefore also for the localization $s M_{h c}$ (this follows from arguments of Hirschhorn $[\mathrm{H}]$ ). Because of this, Proposition 3.2 (b) lets us prove SM7(iii) by checking that if $Z$ is fibrant in $s M_{h c}$ and $K \stackrel{\sim}{\hookrightarrow} L$ is a trivial cofibration of simplicial sets, then $Z^{L} \rightarrow Z^{K}$ is a weak equivalence. Since $Z$ is a simplicial resolution, 
Proposition 4.8 shows that $Z^{L} \rightarrow Z^{K}$ is actually an objectwise weak equivalence (and hence a hocolim-equivalence).

This completes the proof of Theorem 1.2

Remark 6.3. The fact that $s M_{h c}$ is a simplicial model category of course translates into a simple statement about homotopy colimits. To see this, recall that any $X \in s M$ may be regarded as a diagram $X: \Delta^{o p} \rightarrow M$, and this diagram has a homotopy colimit. SM7(iii) for $s M_{h c}$ is the statement that if $A \longmapsto B$ is a Reedy cofibration inducing a weak equivalence between the corrected homotopy colimits, then $(A \otimes L) \coprod_{A \otimes K}(B \otimes K) \rightarrow B \otimes L$ also induces a weak equivalence on corrected homotopy colimits. It would be nice to have a proof of this for arbitrary model categories $M$, but at the moment we don't know of one. It is known to be true if $M$ has the property that a disjoint union of weak equivalences is a weak equivalence, but this does not hold in general. The problem is that in forming $A \otimes K, A \otimes L$, etc., one is forming a bunch of coproducts, and this is not necessarily a good operation. When one goes to compute the correct homotopy colimit of such a diagram, the first step is to replace every object by a cofibrant one; the problem is that a coproduct of cofibrant replacements will not necessarily be the cofibrant replacement of the coproduct. The fact that $A \longmapsto B$ is a Reedy cofibration should save the day, but it is not so clear how to make use of this.

In our situation we really cheated: we side-stepped the whole issue by making use of left properness (which we had to assume anyway) and by milking the existence of a model structure. Somehow the argument feels a little unsatisfying because of this. It does show the power of the model category machinery, however, in the sense that we were able to prove a non-trivial result about homotopy colimits without having to work very hard. This suggests that the model category $s M_{h c}$ may be useful for other kinds of applications.

\section{Some ADDitional REMARKS}

In this final section we raise some open questions related to the above results.

7.1. The Reedy hocolim structure. Let $M$ be a model category of type (LpCe) or $(\mathrm{LpCo})$, and let $\mathcal{C}$ be a contractible Reedy category. Theorem 5.7 gives a model structure on $M^{\mathcal{C}}$ in which

(i) the weak equivalences are the hocolim-equivalences;

(ii) the cofibrations are the Reedy cofibrations;

(iii) the fibrations are the maps with the appropriate right-lifting-property.

Notice that the definitions of these classes make no use at all of our assumptions on $M$. It is therefore natural to wonder whether such a model category structure exists in general.

A first step in this direction is to try to identify the fibrations, and there turns out to be a candidate for what they should be: these are the Reedy fibrations $X \rightarrow Y$ with the property that for every $c \rightarrow d$ in $\mathcal{C}$, the square

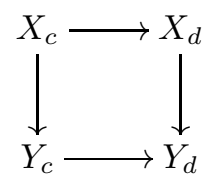

is a homotopy pullback square. 
If the above hocolim-structure exists, it must have the property that a fibration which is a hocolim-equivalence is actually an objectwise weak equivalence. This is because the trivial fibrations of the hocolim-structure are exactly the class of Reedy trivial fibrations, as they are both maps having the right-lifting-property with respect to Reedy cofibrations. We can therefore ask - as a reality check - if the above candidate for the hocolim-fibrations has this property. Unfortunately this question is somewhat awkward; the following is a more manageable question which would imply it:

Question: Let $X$ and $Y$ be diagrams of cofibrant objects. If $X \rightarrow Y$ is a Reedy fibration with the property described in (7.1), does it follow that for each $c \in \mathcal{C}$ the diagram

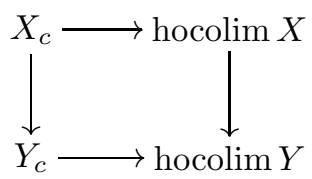

is also a homotopy pullback square?

Remark 7.2. Very loosely stated, condition (7.1) states that the 'difference' between $X_{c}$ and $Y_{c}$ remains constant as $c$ is allowed to vary. The above question asks whether this must also then be the 'difference' between hocolim $X$ and hocolim $Y$.

Note the following observations:

(i) If the answer is yes, this would be a generalization of Chachólski and Scherer's theorem (5.4). When $Y$ is the constant diagram whose value is a point, the question asks: if $X$ is Reedy fibrant with the property that every map is a weak equivalence, does it necessarily follow that $X_{c} \rightarrow \operatorname{hocolim} X$ is an equivalence for every $c \in \mathcal{C}$ ? The Chachólski-Scherer theorem says that the answer is yes.

(ii) An affirmative answer would imply that a Reedy fibration with the property (7.1) which is also a hocolim-equivalence, is in fact an objectwise weak equivalence.

(iii) An affirmative answer would possibly allow one to prove by hand that the hocolim-structure exists, at least under mild hypotheses like cofibrant-generation. In other words, the model category could be constructed without having to localize.

Now we come to the sad truth of the situation, which is that, at the moment, the answer to the above question seems likely to be "no". Even examples of simple indexing categories like the pushout category

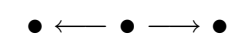

make the result seem questionable. However, the answer does appear to be "yes" in a variety of circumstances. Rezk has proven similar results for model categories coming from simplicial topoi in [R]. Rezk, Schwede, and Shipley [RSS have shown that the result holds when $\mathrm{C}=\Delta^{o p}$ and the model category $M$ is cofibrantlygenerated and stable.

All of this shows the extreme power of the localization machinery, which allowed us to construct the model category without ever understanding what the fibrations are. Although it would be interesting to see some kind of characterization of the fibrations. 
7.3. Replacing model categories by simplicial ones. After being exposed to the results of sections 5 and 6 , the reader may be tempted to conclude that having the hocolim-equivalences among the weak equivalences is in some sense the 'smallest price to pay' for having the model category on $s M$ be simplicial. This is in some sense true, and in some sense false.

Consider the following properties of a model structure on $s M$ :

(i) The categorical simplicial action makes it into a simplicial model category;

(ii) The natural adjunction $c_{*}: M \rightleftarrows s M: e v_{0}$ is a Quillen pair;

(iii) The above adjunction is a Quillen equivalence.

It is possible to see that:

- If a model structure on $s M$ satisfies (i) and (ii), then the identity maps must give a Quillen pair

$$
L: s M_{\text {Reedy }} \rightleftarrows s M: R .
$$

(In particular, every Reedy cofibration must be a cofibration in $s M$.) Moreover, one can show that every fibrant object in $s M$ is a simplicial resolution. It follows that if $M$ is of type (LpCe) or ( $\mathrm{LpCo}$ ), then the identity functors give a Quillen pair

$$
L: s M_{h c} \rightleftarrows s M: R .
$$

- If (iii) is also satisfied, then the above Quillen pair must be a Quillen equivalence between $s M_{h c}$ and $s M$.

What all this says is that in a certain sense the hocolim-structure really is the closest model category to the Reedy structure having properties (i)-(iii) (closest from the right, in any case). However, it may be that the hocolim-structure does not exist in general, but that there is another (Quillen equivalent) model structure which does exist in general. This would be an interesting topic for further research.

As soon as one is willing to forgo both conditions (ii) and (iii) above, it is possible to find simplicial model category structures on $s M$ which are very different than our hocolim-structure. For example, when $M$ is the category $\mathcal{T}_{o p_{*}}$ of pointed topological spaces, there is the $E_{2}$-model structure on $s M$ developed in [DKS]. This is simplicial, and there are more hocolim-equivalences than $E_{2}$-weak equivalences.

7.4. Dual results for cosimplicial objects. It turns out that the category of cosimplicial objects $c M$ also has a natural simplicial action, which is completely dual to the one we have considered on $s M$. We mention that there is at least the possibility of finding a model structure on $c M$ with the properties that

(a) the categorical action makes it a simplicial model category;

(b) the obvious adjoint pair $e v_{0}: c M \rightleftarrows M: c^{*}$ is a Quillen equivalence.

Moreover, a natural candidate for such a model structure arises by dualizing the hocolim-structure on $s M$. That is to say, we can ask to make $c M$ into a model category by defining the

(i) weak equivalences to be the holim-equivalences;

(ii) fibrations to be the Reedy fibrations;

(iii) cofibrations to be the maps with the appropriate left-lifting-property.

At the moment there are no interesting cases where we can actually construct such a model category, however. Techniques dual to those in this paper would allow the model category to be constructed by colocalizing a certain set of maps, but for 
colocalizations there is at the moment no analogue for the theories of Hirschhorn and Smith. There is no common class of model categories for which colocalizations are known to exist.

\section{Appendix A. Some Facts about Model Categories}

The reader should regard this section as a supplement to the book of Hirschhorn $[\mathrm{H}]$. The purpose is to establish some new (but elementary) results about model categories which are needed in the body of the paper. These are Corollary A.2 and Proposition A.5.

Let $M$ be a model category. If $A \in M$ and $Z_{*} \in s M$, let $M\left(A, Z_{*}\right)$ denote the simplicial set obtained by applying the functor $M(A,-)$ levelwise to $Z_{*}$. Note that if $Z_{*}$ is Reedy fibrant, then there is a natural map $\pi_{0} M\left(A, Z_{*}\right) \rightarrow \mathrm{Ho}_{M}\left(A, Z_{0}\right)$ (where the latter denotes maps in the homotopy category of $M)$. This an isomorphism when $A$ is cofibrant, but not in general. Given two maps $f, g: A \rightarrow Z_{0}$, we will write $f \simeq g\left(\right.$ wrt $\left.Z_{*}\right)$ if $f$ and $g$ are in the same path component of $M\left(A, Z_{*}\right)$.

The following result is an easy generalization of something Hirschhorn proves about simplicial model categories.

Proposition A.1. Let $f: A \rightarrow B$ be a map in $M$ and suppose that for every simplicial resolution $Z_{*}$, the induced map $f^{*}: M\left(B, Z_{*}\right) \rightarrow M\left(A, Z_{*}\right)$ yields an isomorphism on $\pi_{0}$. Then $f$ is a weak equivalence in $M$.

Proof. Pick Reedy simplicial resolutions $\hat{A}_{*}$ and $\hat{B}_{*}$ of $A$ and $B$ for which the maps

$$
j_{A}: c_{*} A \rightarrow \hat{A}_{*} \quad \text { and } \quad j_{B}: c_{*} B \rightarrow \hat{B}_{*}
$$

are Reedy trivial cofibrations. The proof will show that it is enough just to know that the maps $M\left(B, \hat{A}_{*}\right) \rightarrow M\left(A, \hat{A}_{*}\right)$ and $M\left(B, \hat{B}_{*}\right) \rightarrow M\left(A, \hat{B}_{*}\right)$ induce isomorphisms on $\pi_{0}$.

The idea is to show that $f$ becomes an isomorphism in the homotopy category. Using the isomorphism $\pi_{0} M\left(B, \hat{A}^{*}\right) \rightarrow \pi_{0} M\left(A, \hat{A}_{*}\right)$ we see that there is a map $g: B \rightarrow \hat{A}_{0}$ projecting onto $j_{A}: A \stackrel{\sim}{\longrightarrow} \hat{A}_{0}$. In other words, there is a $g$ for which $g f \simeq j_{A}\left(\right.$ wrt $\left.\hat{A}_{*}\right)$, and this implies that $g f=j_{A}$ in the homotopy category. But $j_{A}$ was a weak equivalence, so this may be written as $j_{A}^{-1} g \circ f=i d_{A}$ in Ho $M$. The next step is to show that the other composite is also the identity.

Now $f: A \rightarrow B$ lifts to a map $\hat{f}: \hat{A}_{*} \rightarrow \hat{B}_{*}$. It is easy to check that the maps $\hat{f}_{0} g, j_{B}: B \rightarrow B_{0}$ both project onto $\hat{f}_{0} j_{A}$ under the map $\pi_{0} M\left(B, \hat{B}_{*}\right) \rightarrow$ $\pi_{0} M\left(A, \hat{B}_{*}\right)$. Since our assumption is that this is an isomorphism, it follows that $\hat{f}_{0} g \simeq j_{B}\left(\right.$ wrt $\left.\hat{B}_{*}\right)$. This means that $\hat{f}_{0} \circ g=j_{B}$ as maps in the homotopy category. Now the square

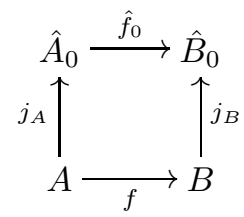

gives that $f \circ j_{A}^{-1}=j_{B}^{-1} \circ \hat{f}_{0}$ in Ho $M$, and therefore

$$
f \circ j_{A}^{-1} g=j_{B}^{-1} \hat{f}_{0} g=j_{B}^{-1} j_{B}=i d_{B} .
$$

This completes the proof that $f$ becomes an isomorphism in Ho $M$, which says that $f$ is a weak equivalence. 
Corollary A.2. Let $M$ and $N$ be model categories, and let $L: M \rightleftarrows N: R$ be an adjoint pair. Then these are Quillen functors if and only if $R$ preserves both fibrations between fibrant objects and trivial fibrations.

There is of course a dual version of this corollary, where $L$ is required to preserve cofibrations between cofibrant objects and trivial cofibrations.

Proof. The 'only if' part is classical, so we only prove the other direction. Our proof depends heavily on adjointness.

The assumption that $R$ preserves trivial fibrations implies that $L$ preserves cofibrations, and what we must show is that $L$ also preserves trivial cofibrations. So let $A \stackrel{\sim}{\sim} B$ be a trivial cofibration. Proposition A.1 lets us check that $L A \rightarrow L B$ is a weak equivalence by examining

$$
N\left(L B, Z_{*}\right) \rightarrow N\left(L A, Z_{*}\right)
$$

for every simplicial resolution $Z_{*} \in s N$. By adjointness, this map is isomorphic to

$$
M\left(A, R Z_{*}\right) \rightarrow M\left(B, R Z_{*}\right) .
$$

Since $A \rightarrow B$ is a trivial cofibration, the above map will be a weak equivalence as long as we can show that $R Z_{*}$ is Reedy fibrant in $s M$. The $n$th matching map of $R Z_{*} \rightarrow *$ may be identified with $R Z_{n} \rightarrow R\left(M_{n} Z\right)$ (using the fact that $R$ commutes with limits). Then the fact that $Z_{*}$ was Reedy fibrant implies that $Z_{n} \rightarrow M_{n} Z$ is a fibration between fibrant objects, and so our assumption that $R$ preserves such maps gives directly that $R Z_{*}$ is also Reedy fibrant.

Remark A.3. The above corollary is especially useful in the context of localizations $S^{-1} M$. The fibrations in a localization are often mysterious, but the trivial fibrations and the fibrations between fibrant objects are the same as those in $M$.

Corollary A.4. Let $f: A \longmapsto B$ be a cofibration between cofibrant objects. Then $f$ is a weak equivalence if and only if $f$ has the left-lifting-property with respect to fibrations between fibrant objects.

Proof. Suppose that $f$ has the stated lifting property. Using the proposition, we only have to show that $M\left(B, Z_{*}\right) \rightarrow M\left(A, Z_{*}\right)$ induces an isomorphism on $\pi_{0}$ for every simplicial resolution $Z_{*}$. Surjectivity is immediate from the assumption on $f$; in fact, $M\left(B, Z_{*}\right) \rightarrow M\left(A, Z_{*}\right)$ is surjective on 0 -simplices. So let $g, h: B \rightarrow Z_{0}$ be maps with the same image in $\pi_{0} M\left(A, Z_{*}\right)$. Since $A$ is cofibrant and $Z_{*}$ fibrant, $M\left(A, Z_{*}\right)$ is a fibrant simplicial set. The fact that $g f$ and $h f$ are in the same path component implies that there is an $H: A \rightarrow Z_{1}$ giving a homotopy between them. In other words, there is a square

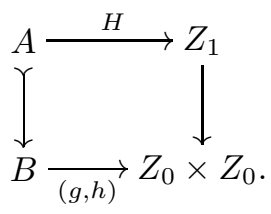

But $Z_{1} \rightarrow Z_{0} \times Z_{0}$ is a Reedy fibration between fibrant objects, and so our assumption on $f$ gives a lift. That is, we get a homotopy between the maps $g, h: B \rightarrow Z_{0}$, which shows they become identified in $\pi_{0} M\left(B, Z_{*}\right)$. This completes the proof. 
The next result runs along very different lines. Recall that in topological spaces and simplicial sets one can detect whether a map is a weak equivalence by examining the function complexes from certain basic objects (e.g., the spheres); in these classical cases this is essentially the definition of weak equivalence. The proposition below gives an analog for 'nice enough' model categories. This is a key element for our construction of the hocolim model structure in section 3 .

Proposition A.5. Let $M$ be a left proper, cofibrantly-generated model category. Then there exists a set $W$ of cofibrant objects which detect weak equivalences, in the sense that a map $X \rightarrow Y$ is a weak equivalence precisely when the induced map of function complexes

$$
\underline{\mathcal{M}}(A, X) \rightarrow \underline{\mathcal{M}}(A, Y)
$$

is a weak equivalence for every $A \in W$.

Proof. Let $\left\{A_{\alpha} \rightarrow B_{\alpha}\right\}$ be a set of generating cofibrations, and let $\tilde{A}_{\alpha} \longmapsto \tilde{B}_{\alpha}$ denote cofibrations between cofibrant objects which are weakly equivalent to the $A_{\alpha} \rightarrow B_{\alpha}$. Define $W$ to be $\left\{\tilde{A}_{\alpha}, \tilde{B}_{\alpha}\right\}$.

Suppose that $X \rightarrow Y$ induces weak equivalences on the function complexes obtained by mapping from elements of $W$. Let $\hat{X}_{*} \rightarrow \hat{Y}_{*}$ be a Reedy fibration between fibrant objects which is a replacement for $c_{*} X \rightarrow c_{*} Y$ in the Reedy model category $s M$. To show that $X \rightarrow Y$ is a weak equivalence, it is enough to show that $M\left(C, \hat{X}_{*}\right) \rightarrow M\left(C, \hat{Y}_{*}\right)$ is a weak equivalence of simplicial sets for every cofibrant object $C \in M$. Our assumption yields that this map is a weak equivalence when $C$ is one of the $\tilde{A}_{\alpha}$ or $\tilde{B}_{\alpha}$, and so the idea will be to build an arbitrary $C$ (up to homotopy) from these basic objects. Cofibrant-generation says that any $C$ may be built up from the $A_{\alpha}$ and $B_{\alpha}$, but this is not quite what we want. This is why we have to assume left properness.

Recall that the small object argument gives us a (possibly transfinite) sequence of spaces

$$
\emptyset=C_{0} \rightarrow C_{1} \rightarrow C_{2} \rightarrow \cdots \quad \Rightarrow C_{\infty}
$$

with the properties that

(i) $C_{\infty}=\operatorname{colim}_{n} C_{n}$;

(ii) $C$ is a retract of $C_{\infty}$;

(iii) $C_{n+1}$ is obtained from $C_{n}$ by pushing-out along the maps $A_{\alpha} \longmapsto B_{\alpha}$.

The argument now proceeds in several steps:

Step 1. Each $C_{n}$ induces a weak equivalence $M\left(C_{n}, X_{*}\right) \rightarrow M\left(C_{n}, Y_{*}\right)$.

This is trivial for $C_{0}$, and so we assume the statement for $C_{n-1}$ and proceed by induction using the fact that $C_{n}$ sits in a pushout diagram

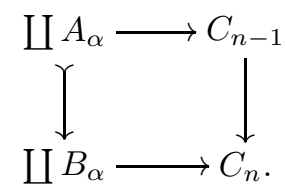


By a result of $[\mathrm{H}]$, left properness guarantees that there are cofibrant approximations $\tilde{C}_{n-1}$ and $\tilde{C}_{n}$ which sit in a pushout square

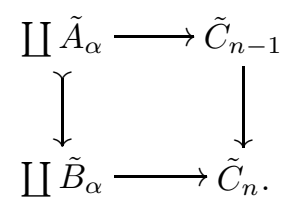

Mapping into $X_{*}$ and $Y_{*}$ gives a diagram

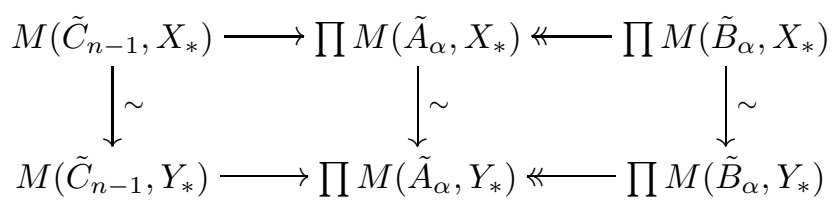

where the pullback of the horizontal rows are $M\left(\tilde{C}_{n}, X_{*}\right)$ and $M\left(\tilde{C}_{n}, Y_{*}\right)$. The vertical maps are weak equivalences by the assumption about $X \rightarrow Y$ and the induction hypothesis (and this is where we really need $\tilde{A}$ and $\tilde{B}$ instead of $A$ and $B$, since $M\left(A, X_{*}\right)$ and $M\left(B, X_{*}\right)$ need not be homotopy function complexes). The indicated maps are fibrations because $\tilde{A}_{\alpha} \rightarrow \tilde{B}_{\alpha}$ are cofibrations and $X_{*}$ and $Y_{*}$ are Reedy fibrant. This all implies that the induced map between the pullbacks is a weak equivalence of simplicial sets (using the fact that $s \mathcal{S}$ et is right proper, if you wish). This is equivalent to the statement that $M\left(C_{n}, X_{*}\right) \rightarrow M\left(C_{n}, Y_{*}\right)$ is a weak equivalence, using the fact that $\tilde{C}_{n} \rightarrow C_{n}$ is a weak equivalence between cofibrant objects.

Step 2. $C_{\infty}$ induces a weak equivalence $M\left(C_{\infty}, X_{*}\right) \rightarrow M\left(C_{\infty}, Y_{*}\right)$.

Since $C_{\infty}$ is the colimit of the $C_{n}$, the simplicial set $M\left(C_{\infty}, X_{*}\right)$ is the limit of the $M\left(C_{n}, X_{*}\right)$. The maps $M\left(C_{n}, X_{*}\right) \rightarrow M\left(C_{n}, Y_{*}\right)$ are fibrations because $C_{n}$ is cofibrant and $X_{*} \rightarrow Y_{*}$ is a Reedy fibration, and they are weak equivalences by Step 1. We now use the fact that a (possibly transfinite) sequential inverse limit of trivial fibrations is again a trivial fibration.

Step 3. $C$ induces a weak equivalence $M\left(C, X_{*}\right) \rightarrow M\left(C, Y_{*}\right)$.

Since $C$ is a retract of $C_{\infty}$, the map in question is a retract of $M\left(C_{\infty}, X_{*}\right) \rightarrow$ $M\left(C_{\infty}, Y_{*}\right)$, which was just shown to be a weak equivalence. A retract of a weak equivalence is again a weak equivalence.

Thus, we have shown that any cofibrant object yields a weak equivalence of mapping spaces to $X_{*} \rightarrow Y_{*}$. This is known to imply that $X \rightarrow Y$ is a weak equivalence in $M$ (as always, see $[\mathrm{H}]$ ).

\section{REFERENCES}

[B] F. Borceux, Handbook of categorical algebra II, Categories and structures, Cambridge University Press, 1994. MR 96g:18001b

[BK] A.K. Bousfield and D.M. Kan, Homotopy limits, completions, and localizations, Springer Lecture Notes in Math. 304, Springer-Verlag, New York, 1972. MR 51:1825

[CS] W. Chachólski and J. Scherer, Homotopy meaningful constructions: Homotopy colimits, 1998, preprint.

[DHK] W. G. Dwyer, P. S. Hirschhorn, D. M. Kan, Model categories and more general abstract nonsense, in preparation.

[DKS] W. G. Dwyer, D. M. Kan, C. R. Stover, An $E_{2}$ model category structure for pointed simplicial spaces, J. Pure Appl. Alg. 90 (1993), pp. 137-152. MR 95c:55027 
[H] P. S. Hirschhorn, Localization of Model Categories, 1998 preprint.

(Available at http://www-math.mit.edu/ psh).

[Ho] M. Hovey, Model Categories, Mathematical Surveys and Monographs vol. 63, Amer. Math. Soc., 1999. MR 99h:55031

[McL] S. Mac Lane, Categories for the Working Mathematician, Grad. Texts in Math., Vol. 5, Springer-Verlag, New York, 1971. CMP 2000:01

[Q] D. Quillen, Homotopical Algebra, Springer Lecture Notes in Math. 43, Springer-Verlag, Berlin, 1967. MR 36:6480

[R] C. Rezk, Fibrations and homotopy colimits of simplicial presheaves, 1998 Preprint.

[RSS] C. Rezk, S. Schwede, and B. Shipley, Simplicial Model Categories, in preparation.

[S] J. Smith, Combinatorial model categories, in preparation.

Department of Mathematics, Purdue University, West Lafayette, Indiana 47907

E-mail address: ddugger@math.purdue.edu 